\title{
A Study on the Manufacturing Properties of Crack Self-Healing Capsules Using Cement Powder for Addition to Cement Composites
}

\author{
Yun-Wang Choi, Sung-Rok Oh, and Byung-Keol Choi \\ Department of Civil Engineering, Semyung University, Jecheon 390-711, Republic of Korea \\ Correspondence should be addressed to Yun-Wang Choi; crete77@semyung.ac.kr
}

Received 21 April 2017; Accepted 22 August 2017; Published 1 October 2017

Academic Editor: Xiao-Yong Wang

Copyright (C) 2017 Yun-Wang Choi et al. This is an open access article distributed under the Creative Commons Attribution License, which permits unrestricted use, distribution, and reproduction in any medium, provided the original work is properly cited.

\begin{abstract}
We fabricated crack self-healing capsules using cement powder for mixing into cement composites and evaluated the properties of the capsule manufacturing process in this study. The manufacture of the self-healing capsules is divided into core production processing of granulating cement in powder form and a coating process for creating a wall on the surfaces of the granulated cement particles. The produced capsules contain unhardened cement and can be mixed directly with the cement composite materials because they are protected from moisture by the wall material. Therefore, the untreated cement is present in the form of a capsule within the cement composite, and hydration can be induced by moisture penetrating the crack surface in the event of cracking. In the process of granulating the cement, it is important to obtain a suitable consistency through the kneading agent and to maintain the moisture barrier performance of the wall material. We can utilize the results of this study as a basis for advanced self-healing capsule technology for cement composites.
\end{abstract}

\section{Introduction}

Smart structures and intelligent materials have emerged as new trends for reducing cracks in concrete, and extensive research is being performed on the self-healing of concrete cracks. Self-healing structures can detect early-stage cracks and heal themselves, thereby reducing time and cost involved in maintenance and repair, compared to those of general structures. A major advantage of self-healing is that repairs can be performed easily, even for structures that are difficult to access physically $[1,2]$. Among the various methods enabling self-healing, one approach is to encapsulate materials that have self-healing capabilities and introduce them as admixtures in concrete. Such capsules can hold large amounts of self-healing materials $[3,4]$ and reactions can be selectively induced at cracks. In one international study, self-healing materials and catalysts were encapsulated as microsized capsules $[5,6]$. Meanwhile, in Korea, selfhealing microsized capsules capable of reacting with sunlight or moisture without catalysts have been applied in actual construction [7]. Two conclusions can be derived from these case studies at home and abroad. First, the target of selfhealing capsules is not the structure's matrix, but its surface. Second, materials that have self-healing capabilities can be applied to organic chemical products $[8,9]$. The number of capsules available for self-healing is limited, because they are normally utilized as coatings to protect the concrete surface, which impedes the healing of deep cracks. Because most of the structure's matrix comprises inorganic composites, the self-healing performance can be enhanced by using inorganic materials with similar thermal properties instead of organic materials with varied thermal properties. While directly mixing the cementitious composites, the thickness of the matrix in the capsule can be freely adjusted as necessary. The self-healing performance can also be maximized by introducing more self-healing capsules.

To develop cementitious composites with self-healing capabilities by distributing self-healing capsules within the structure's matrix, this study developed self-healing capsules with the same properties as that of the target cementitious composites, as well as capsules suitable for direct mixing with cementitious composites. The properties of the self-healing 
TABLE 1: Chemical components and physical properties of cement.

\begin{tabular}{|c|c|c|c|c|c|c|c|c|c|}
\hline \multirow[b]{2}{*}{ Types } & \multicolumn{9}{|c|}{ Items } \\
\hline & $\begin{array}{l}\mathrm{CaO} \\
(\%)\end{array}$ & $\begin{array}{r}\mathrm{SiO}_{2} \\
(\%)\end{array}$ & $\begin{array}{c}\mathrm{Al}_{2} \mathrm{O}_{3} \\
(\%)\end{array}$ & $\begin{array}{c}\mathrm{MgO} \\
(\%)\end{array}$ & $\begin{array}{c}\mathrm{Fe}_{2} \mathrm{O}_{3} \\
(\%)\end{array}$ & $\begin{array}{l}\mathrm{SO}_{3} \\
(\%)\end{array}$ & $\begin{array}{c}\text { L.O.I. }^{a} \\
(\%)\end{array}$ & $\begin{array}{l}\text { Surface area } \\
\left(\mathrm{cm}^{2} / \mathrm{g}\right)\end{array}$ & $\begin{array}{l}\text { Density } \\
\left(\mathrm{g} / \mathrm{cm}^{3}\right)\end{array}$ \\
\hline OPC & 61.40 & 21.60 & 3.40 & 2.50 & 3.10 & 2.50 & 0.03 & 3,540 & 3.15 \\
\hline
\end{tabular}

${ }^{\mathrm{a}}$ Loss on ignition.

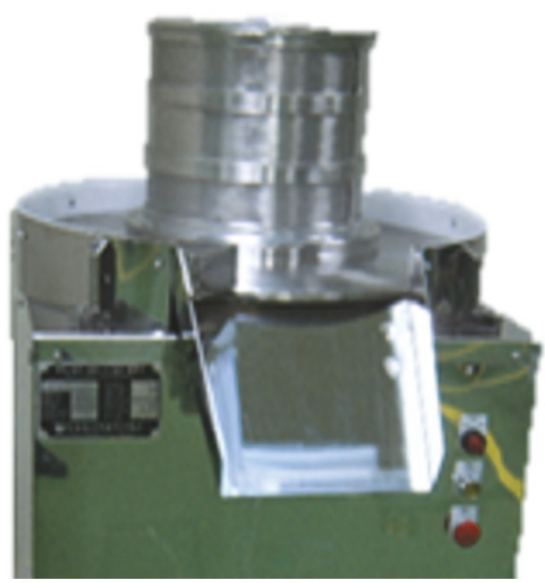

(a) Core production equipment

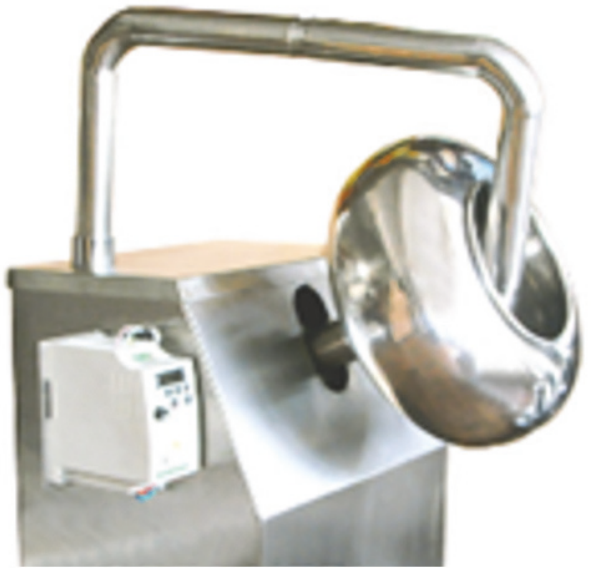

(b) Wall coating equipment

FIGURE 1: Capsule manufacturing device.

capsules were also evaluated. This study did not consider the healing effects of the self-healing capsules.

Instead, it focused on evaluating the core production processes for granulating cement in powder form, the coating process of walls on the surface of the granulated cement particles, and the effects of capsule addition on the quality of the cementitious composites. The results of this study can serve as a valuable reference for developing self-healing capsules and capsule-containing cementitious composites.

\section{Experimental Outline}

\subsection{Fabrication of Self-Healing Capsules}

2.1.1. Core Material. The core material of the self-healing capsules, hereinafter referred to as "SHCs," was common ordinary Portland cement powder, hereinafter referred to as "OPC." Table 1 shows the chemical composition and physical properties of the OPC used as the core material. Since cement generally reacts with water via hydration [10], four high-purity reagents are utilized as kneading agents; they contain little water, provide coagulation, and are easily evaporated. The four reagents used as the kneading agent are methyl alcohol $\left(\mathrm{CH}_{4} \mathrm{O}\right)$, chloroform $\left(\mathrm{CHCl}_{3}\right)$, dichloromethane $\left(\mathrm{CH}_{2} \mathrm{Cl}_{2}\right)$, and acetone $\left(\mathrm{C}_{3} \mathrm{H}_{6} \mathrm{O}\right)$. In order to evaluate the quality of the core material according to the type of kneading agent, the core material utilizing each kneading agent (hereinafter, "KA") was pulverized to measure the density. A 50\% water/cement (W/C) paste was prepared and used to evaluate the compressive strength. The granulated cement powder was processed by a capsule core manufacturing apparatus as dough to prepare the core material. Figure 1(a) shows the capsule core manufacturing equipment used in the experiment.

2.1.2. Wall Material. Because the core material granulated with cement (hereinafter, "core material") can react with water when it is mixed with the cement composite, thereby losing the components of the cement composite and the mixer, three suitable wall materials were selected and tested. The wall materials were liquid rubber ("LR"), an acryl-based material of poly(methyl methacrylate) ("AM"), and silicatebased materials (a mixture of sodium silicate, potassium silicate, and lithium silicate, hereinafter referred to as "SM").

Because $\mathrm{AM}$ is solid in the operating temperature range, dichloromethane $\left(\mathrm{CH}_{2} \mathrm{Cl}_{2}\right.$, hereinafter referred to as "MC") was used as a solubilizer in order to dissolve AM. The performance of each wall material was assessed by applying the test methods specified in KS L 5105 "testing method for compressive strength of hydraulic cement mortars" after standard mortar mixing. Moisture resistance was assessed through the permeability test specified in KS F 4930 "penetrating water repellency of liquid type for concrete surface application," and the strength was evaluated for samples cured for 3 days according to KS L 5105 "testing method for compressive strength of hydraulic cement mortars."

The core material is inserted into the chamber of Figure 1 (b) and the wall material is coated and dried, while the chamber rotates at a certain rate. Figure 2 shows the wall materials of the capsules used in the experiment, and Figure 3 presents the samples used to assess the moisture resistance and strength of the wall material. 


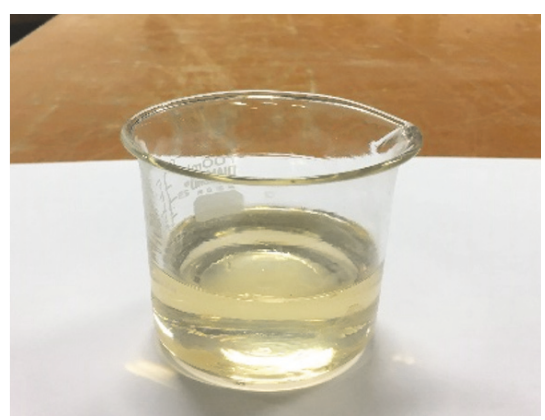

(a) LR

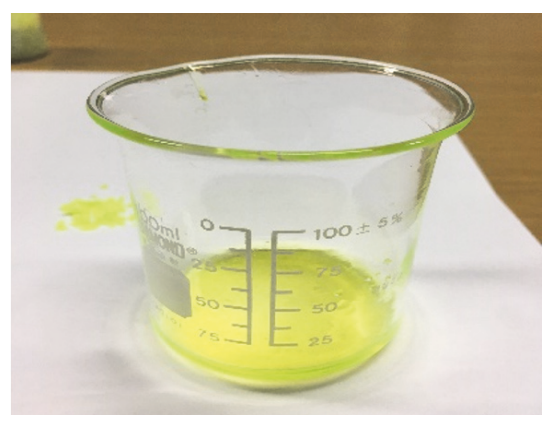

(b) Dissolved AM

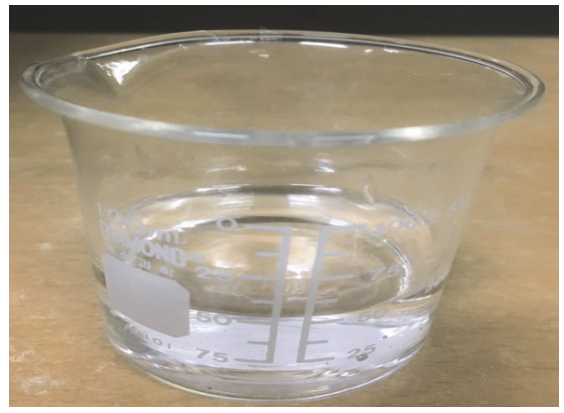

(c) SM

FIGURE 2: Wall materials.

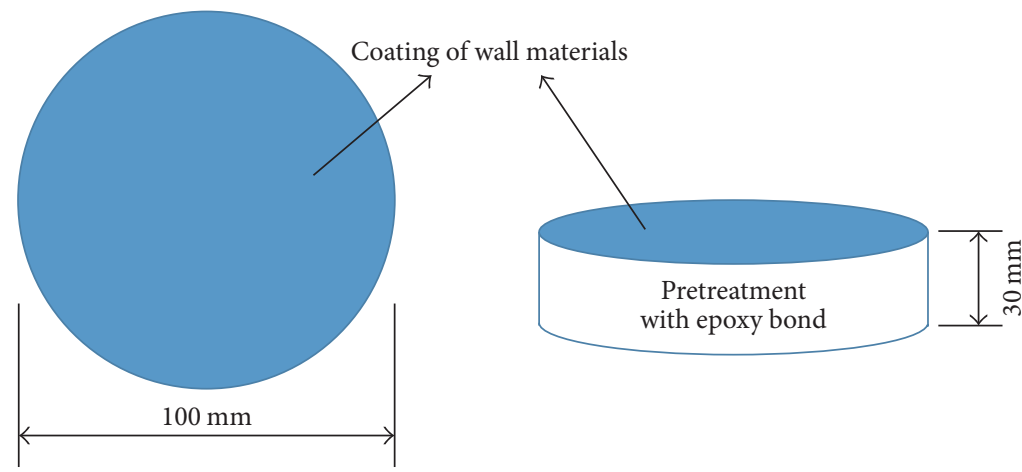

(a) Permeability tests (KS F 4930)

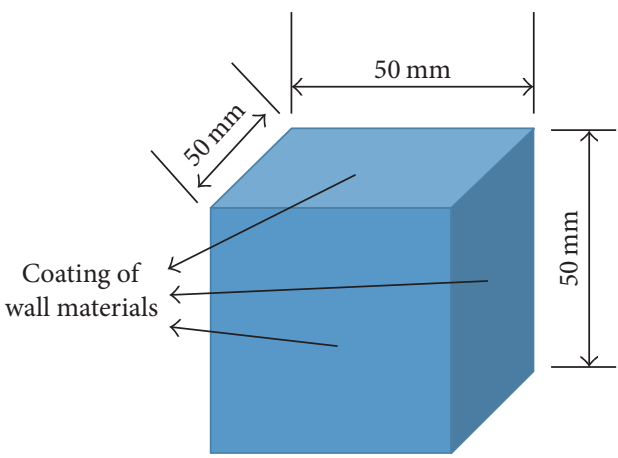

(b) Strength test (KS L 5105)

FIGURE 3: Evaluation methods for wall material.

\subsection{Experimental Method}

2.2.1. Shape. The shape of the core material was magnified by 300 times and observed using a video camera, and the resistance of the capsule shell to moisture was assessed.

2.2.2. Density. To evaluate the effect of the remaining moisture of KA, the cores prepared with each KA type were pulverized and their densities were measured according to KS L 5110 "testing method for specific gravity of hydraulic cement.”

2.2.3. Moisture Resistance. For the moisture resistance of the wall material, a test piece as shown in Figure 3(a) was prepared in accordance with KS F 4930 "penetrating water repellency of liquid type for concrete surface application" and a permeability test was conducted.

2.2.4. Compressive Strength. The compressive strengths of the core materials produced from each KA and the wall materials were measured according to KS L 5105 "testing method for compressive strength of hydraulic cement mortars." Figure 3(b) shows the specimen used for compressive strength testing.

2.2.5. Loss Rate. The loss rate of SHCs was evaluated by measuring the lost mass of SHCs by performing a washing test using the method specified in KS F 2511 "testing method for amount of material finer than $0.08 \mathrm{~mm}$ sieve in aggregate" immediately after mixing the blended mixture of SHCs.

\section{Experimental Results and Discussion}

\subsection{Fabrication of SHCs}

3.1.1. Consistency. SHCs are produced by creating the core material followed by coating. The core material must have a certain consistency for successful processing by the core material fabricator. If the kneading state is too thin or too thick, it is difficult to fabricate the core material. This study utilized OPC as the core material and applied methyl alcohol at three levels (Step 1:10,20, or 30 mass\%) of the OPC mass to obtain the desired consistency. For a quantitative analysis of consistency, a table flow test was performed, and consistency standards for the core materials were established based on the slump values.

Table 2 and Figure 4 give the shapes of Step 1 samples immediately after kneading and the results of the table flow test. The results show that samples with $10 \%$ and $20 \%$ methyl alcohol are easily agglomerated, but the table flow test cannot be performed, as the samples are crushed under very slight shocks. In addition, for the $30 \%$ alcohol mixture, the flow value is obtained rather than the table flow value because the kneading consistency is too watery. 
TABLE 2: Consistencies of Step 1 test group.

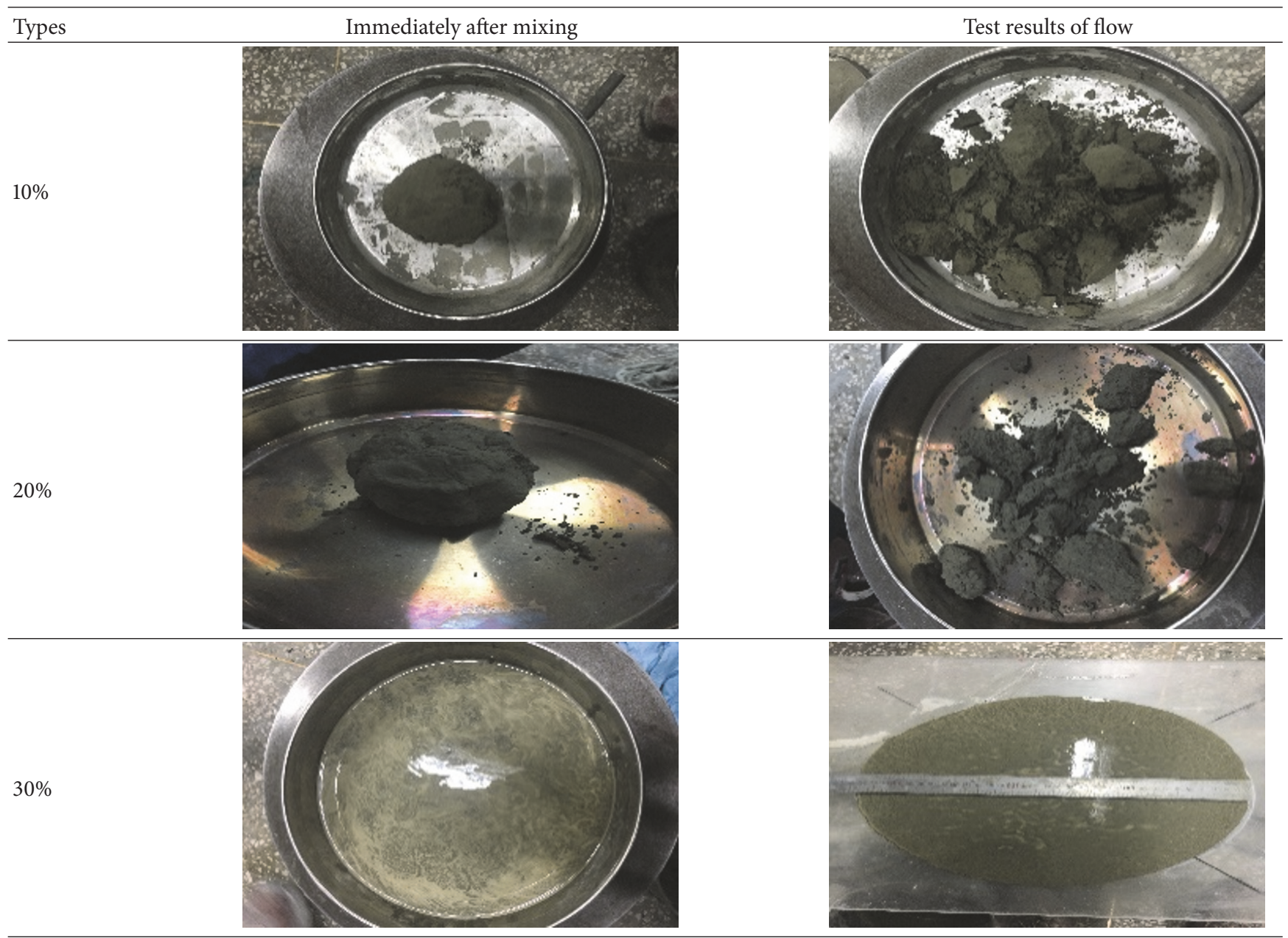

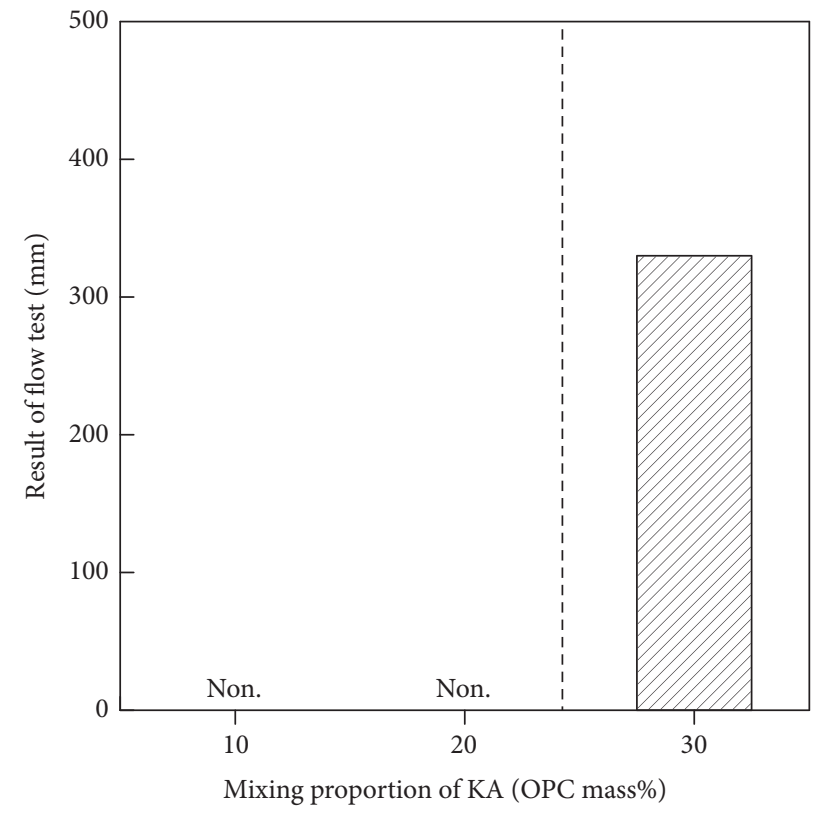

FIGURE 4: Results of flow test for Step 1 group.
The capsule core machine uses the pressure generated by the chamber lid and the mixing blades in the chamber to pass through the micropores on the side walls of the chamber to produce the core material. $10 \%$ and $20 \%$ showed good agglomeration immediately after mixing, but they were easily pulverized when subjected to impact through table flow test. Therefore, kneading states such as those of the $10 \%$ and $20 \%$ methyl alcohol mixtures, which are unable to withstand shocks, are unsuitable, because they are pulverized when the wall material is applied or during drying. Similarly, the kneading state achieved with 30\% methyl alcohol cannot agglomerate because the mixture is too watery after the core material is formed. Therefore, an adequate consistency can be achieved between $20 \%$ and $30 \%$ methyl alcohol by mass. To obtain a suitable consistency, the experiments were repeated with five mixing ratios (Step 2: 21, 22, 23, 24, and 25 mass\%).

Table 3 and Figure 5 show the shapes of Step 2 specimens immediately after kneading and the results of the table flow test. In the case of $21 \%$ methyl alcohol, agglomeration occurs immediately after mixing, but measurements are difficult because the exterior collapses. Samples of $22 \%$ and $23 \%$ alcohol maintain agglomerated forms with slump values 
TABle 3: Consistencies of Step 2 test group.

\begin{tabular}{|c|c|c|}
\hline Types & Immediately after mixing & Test results of flow \\
\hline $21 \%$ & & \\
\hline $22 \%$ & & \\
\hline $23 \%$ & & \\
\hline $24 \%$ & & \\
\hline $25 \%$ & 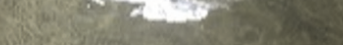 & \\
\hline
\end{tabular}


TABLE 4: Form of produced capsule cores.

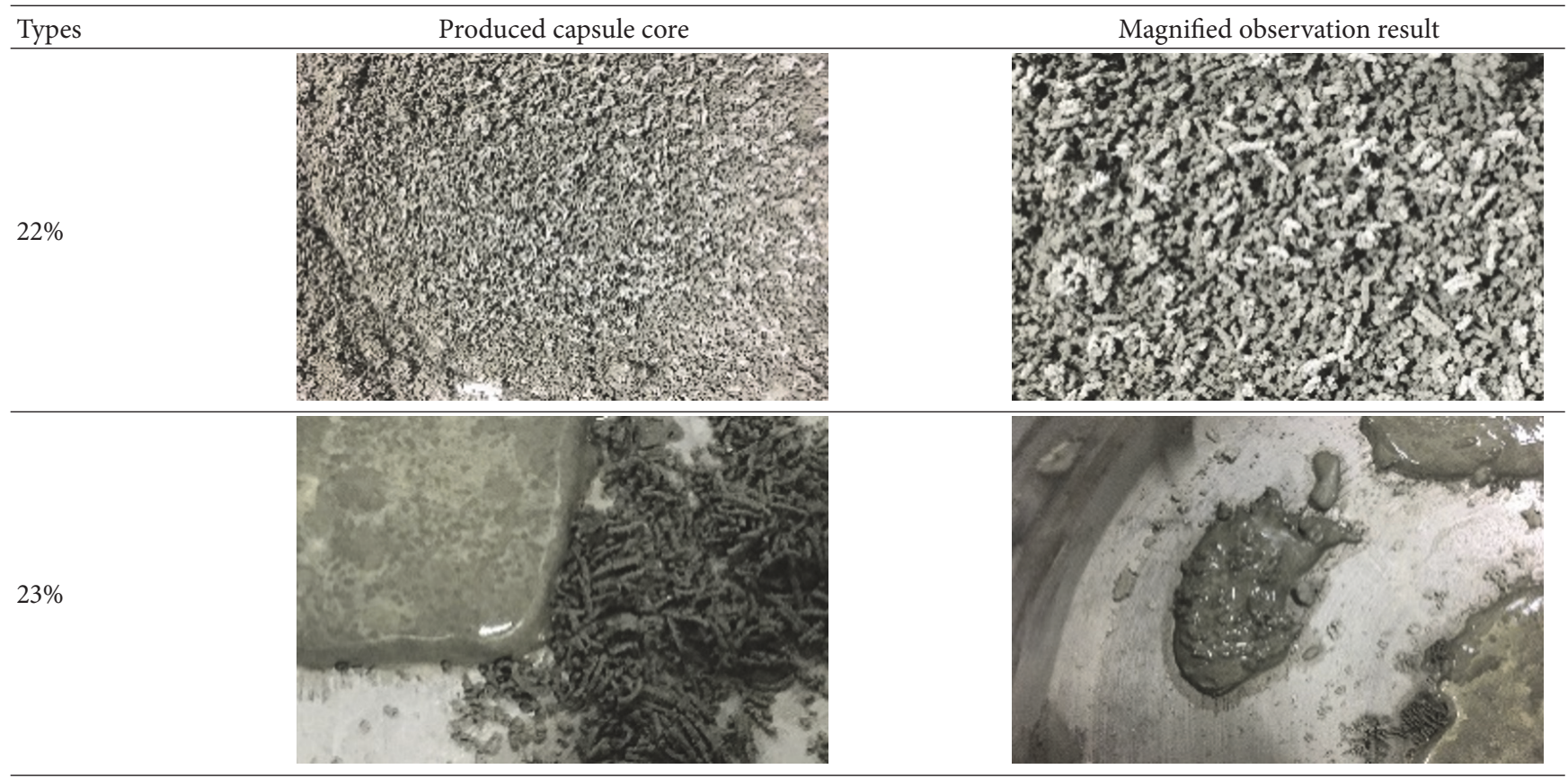

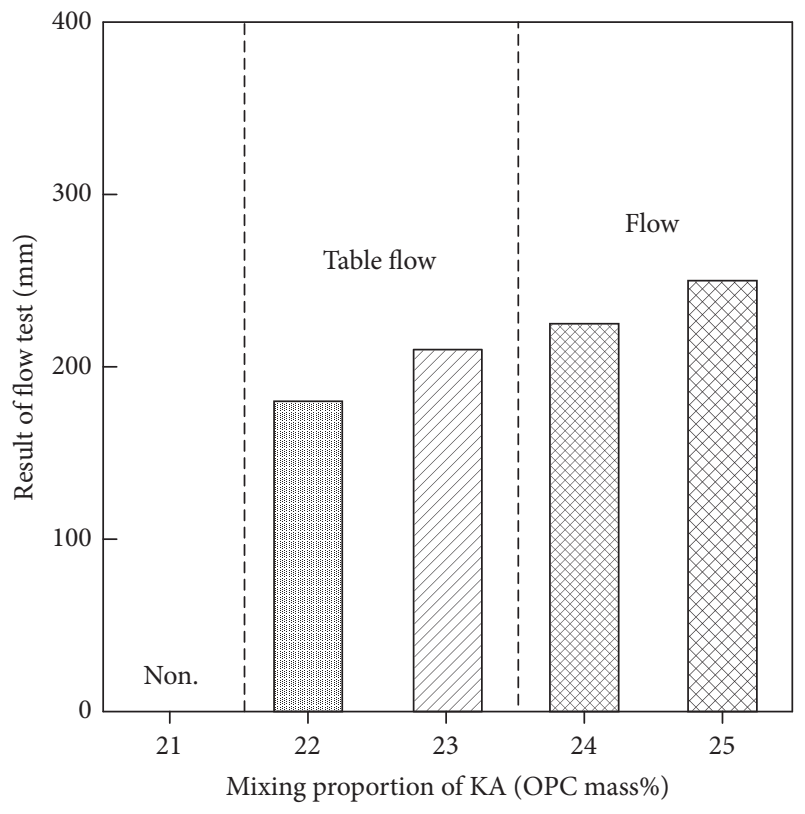

FIGURE 5: Results of flow test for Step 2 group.

obtained based on the table flow results. Similar to the results for the $30 \%$ samples, the samples with $24 \%$ and $25 \%$ methyl alcohol are too watery to become agglomerated, and the flow value is judged to be meaningless. These results indicate that an adequate consistency in creating the core material can be achieved by mixing the KA at 22-23 mass $\%$.

Table 4 and Figure 6 show the production and loss ratio of the core material after mixing the KA at $22 \%$ and $23 \%$.

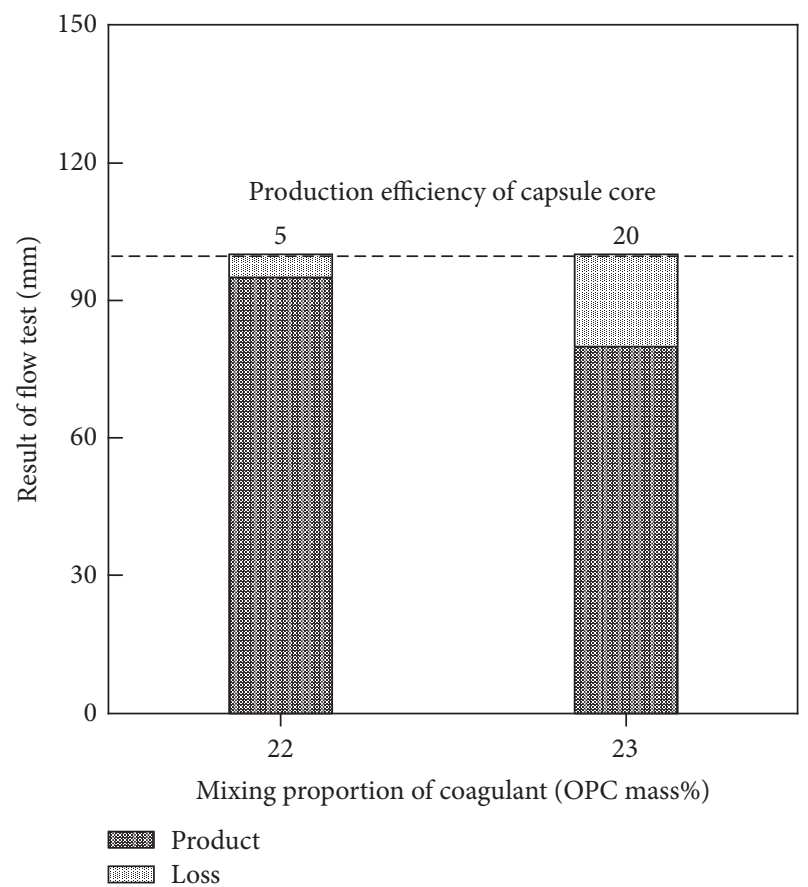

Figure 6: Production efficiency of capsule core.

The results of Table 4 show that $22 \%$ of the particles retained the shape of the particles but $23 \%$ of the particles showed aggregation of the particles during the production process. When examining the loss of core material in Figure 6, samples of $22 \%$ show $5 \%$ losses in mass, while samples of $23 \%$ show segregation of the KA from pressure within the 


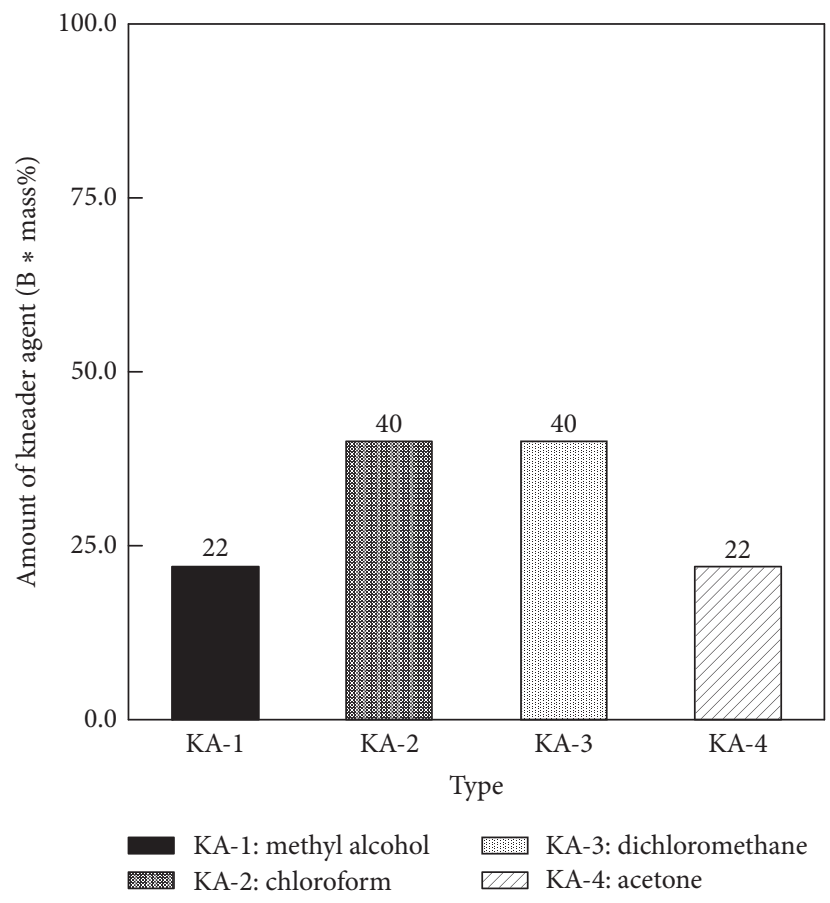

FIgURE 7: Required amount of KA.

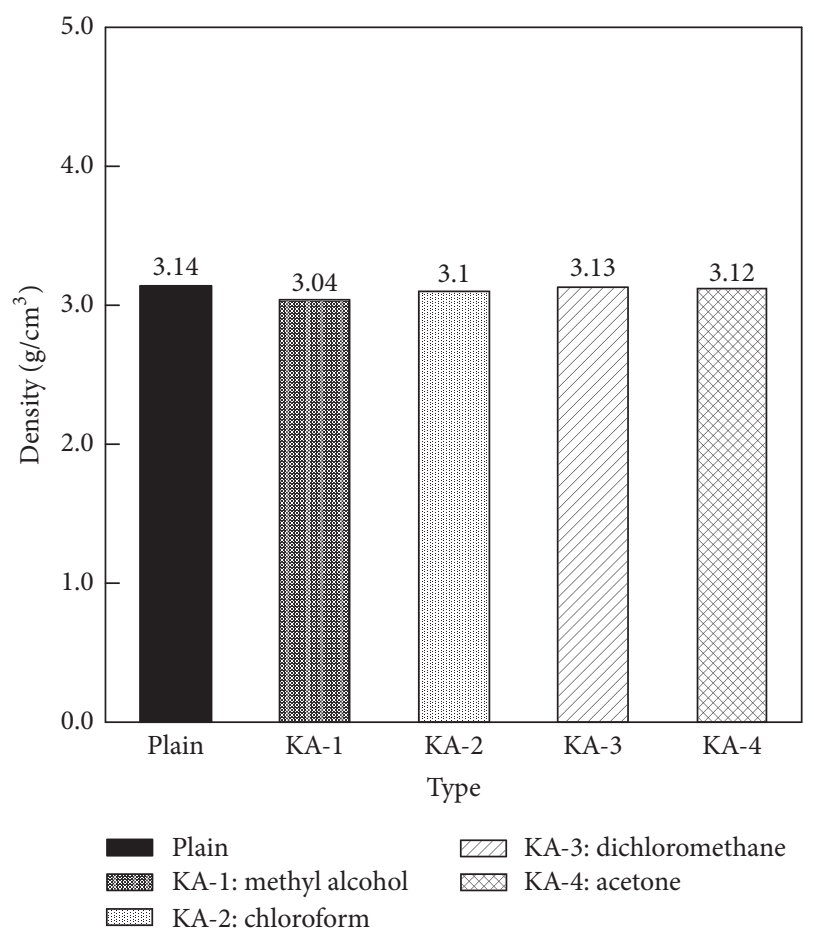

FIGURE 8: Density of core material according to KA type.

The decrease in density can be related to the reactivity of the SHCs; this study focused on evaluating and examining the phenomena of reactivity. While the reason for the decrease in density was not analyzed thoroughly here, the reactivity of the core material is expected to be lower than that of the initial state.

Figure 9 shows the compressive strengths of the paste samples of $50 \% \mathrm{~W} / \mathrm{C}$ using the pulverized core materials made with each KA, as shown in Figure 8. The data marked "plain" represents the paste sample of the OPC without a $\mathrm{KA}$. The compressive strengths of the mixtures with KA1, KA-2, KA-3, and KA-4 tend to decrease by 51, 9.8, 0.1, and $14 \%$ compared to the plain specimen. These results are attributed to the same causes as the density test results, and KA-1 is considered to cause a decrease in strength by approximately half. In addition, the strengths of the KA-2 and KA-4 specimens are decreased by approximately $10 \%$; KA-3 alone does not significantly affect the strength. Therefore, the use of KA-3 is expected to maximize the reactivity of SHCs.

3.1.3. Properties of Wall Materials. Since the core material reacts with moisture, moisture must be blocked from the core material until cracks occur; this way, the core material maintains the activation energy necessary for self-healing. To assess the properties of the wall materials, permeability and compressive strength tests were performed after standard mortar mixing.

Figures 10 and 11 show the results of the permeability and compressive strength tests. The permeation amounts of LR, AM, and SM are smaller than that of the plain sample core material may react with moisture within the constanttemperature and -humidity device when left inside for drying. 


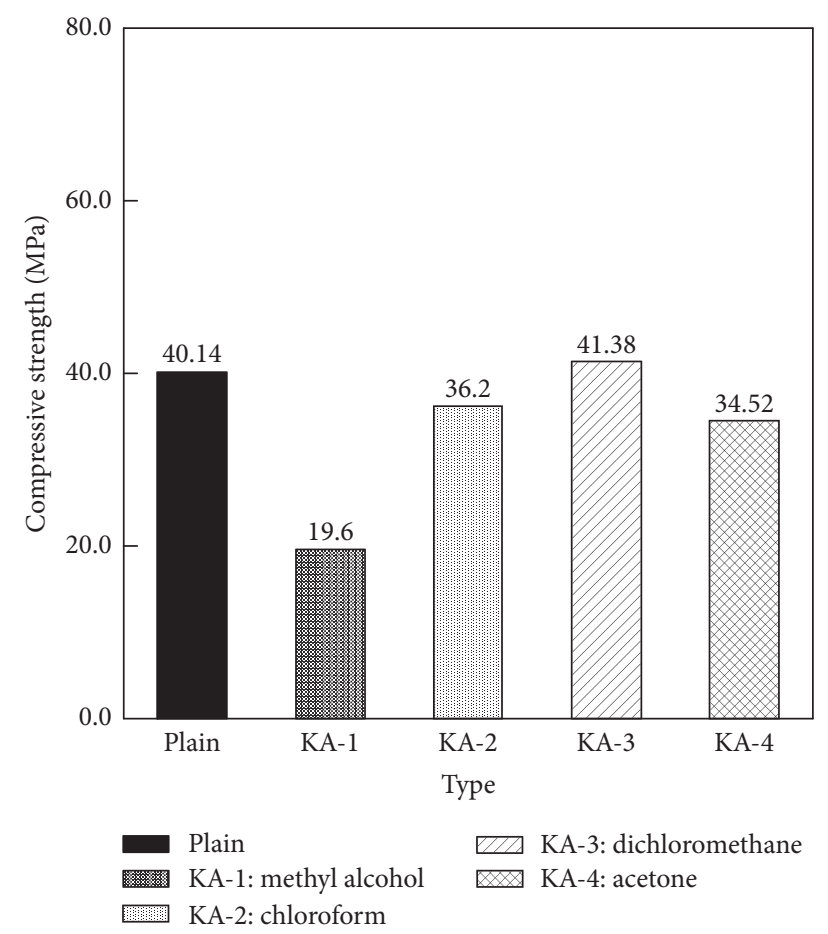

FIGURE 9: Compressive strength of core material according to KA type.

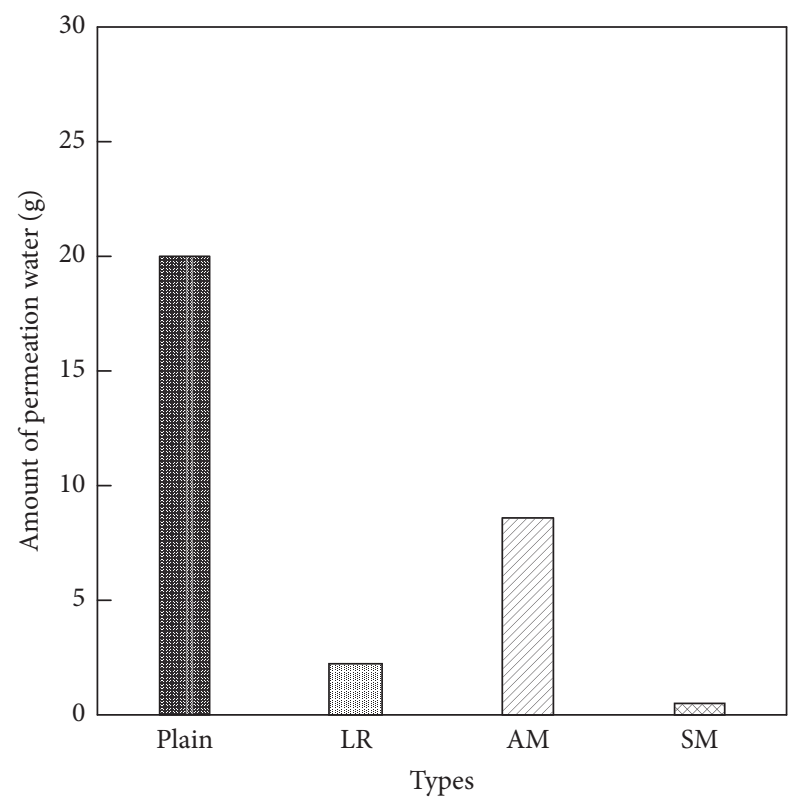

FIGURE 10: Amount of permeation water according to membrane material.

without any wall material by $88 \%, 57 \%$, and $98 \%$, respectively. AM is relatively less permeable than LR and SM are. This can be attributed to the dilution of AM by 20 times in the dissolvent and the decreased uniformity of the dissolvent coating from the relatively high-viscosity AM compared to those of LR and SM. Even for a uniform AM coating, the effective amount may vary depending on the number of layers

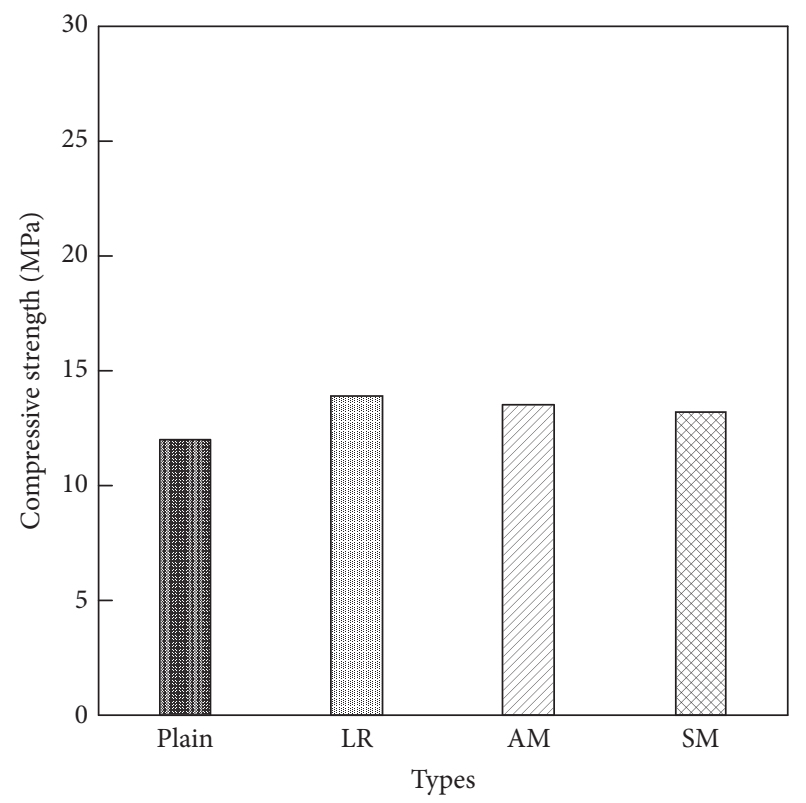

FIgURE 11: Compressive strength according to wall materials.

of the coating and the time elapsed since the solvent of the coated AM has evaporated. The effects of AM dissolution on the effective content and coating require further review through permeability tests.

Figure 11 shows that the compressive strengths of the LR-, AM-, and SM-coated specimens are higher than that of the plain sample by approximately $15.8 \%, 12.7 \%$, and $10.0 \%$, respectively. The strengths of the wall materials tend to increase, improving the surface strength of the core material. In addition, SM is considered to be highly resistant to moisture because it is mainly used as a waterproof material in structures. However, since SM contains a large amount of water, in this study, LR was used as a trial wall material first.

\subsection{Quality of SHCs}

3.2.1. Shape. Figures 12 and 13 show the shapes of the fabricated SHCs. The particle size of SHCs is found to be in the range of $70-1,500 \mu \mathrm{m}$. The particle shapes are spherical, angular, or in the form of cylindrical grains. Most particles are shaped like cylindrical grains because they are fabricated through the holes on the side walls of the pressurized chamber in the core material manufacturing apparatus. In addition, the SHCs are fabricated with wide ranges of particle size, which can be adjusted using the pressure within the chamber or the RPM of the mixing blades. The effect of the particle sizes of the SHCs on the quality of the unhardened cementitious composites and the quality and self-healing effects of the cured composites should be analyzed more thoroughly in the future.

Figure 14 shows the evaluation of the SHCs after applying the wall material. The formation of the capsule shell is qualitatively assessed based on the surface tension. The results of Figure 14 show that moisture is immediately absorbed for samples without wall materials, whereas droplets are formed 


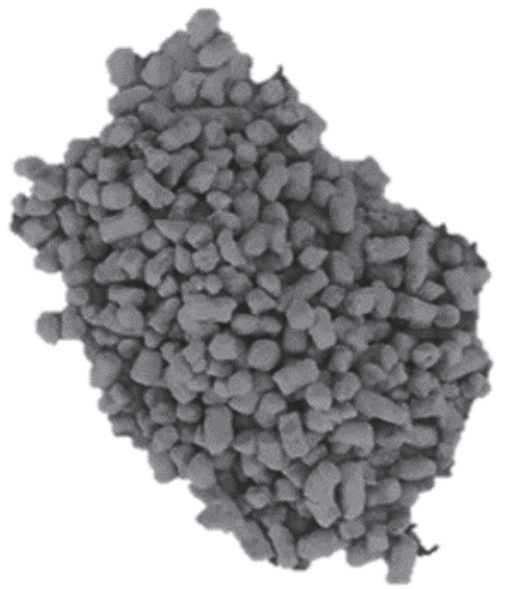

FIGURE 12: Cylindrical shape.

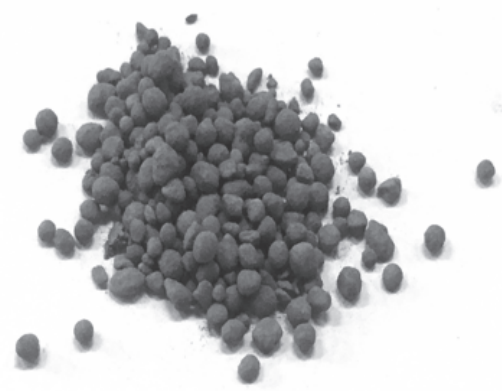

FIGURE 13: Sphere shape.

by the larger surface tension of samples with wall materials. The presence of the wall material can be determined in this manner. Further quantitative analysis may be performed to assess the efficiency of the wall material coating.

3.2.2. Loss Rate. Figures 15 and 16 show the washing tests and losses of SHCs when they are mixed into cement composites. Fresh cement composites containing SHCs were sampled and the remainders, excepting the SHCs, were washed out and dried. As can be seen from Figures 15 and 16, the loss is less than $5 \%$ for the paste after mixing and about 10 to $15 \%$ for the mortar. When the SHCs are mixed with the paste and mortar, they are likely influenced by the mixing blades during mixing. Because mixing with the mortar involves the production of fine aggregates, the rate of loss is increased by two to three times compared to that of the paste. This loss could be compensated by reinforcing the wall material.

\section{Conclusion}

As part of the basic research required to develop cementitious composites with self-healing capabilities, this study fabricated self-healing capsules with cement powders that exhibit the same properties as cementitious composites used

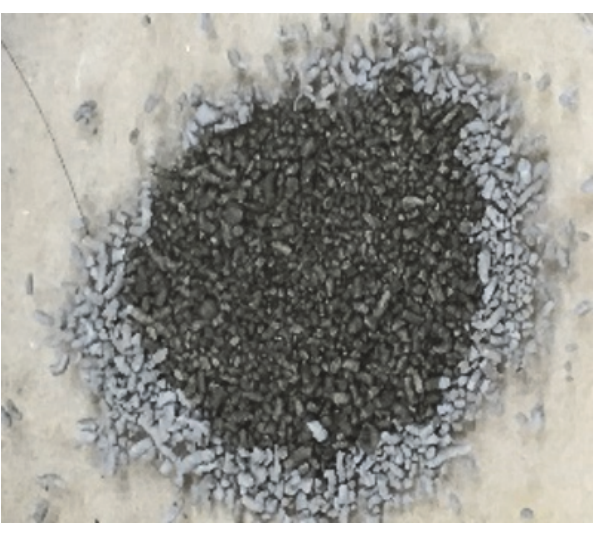

(a) Before coating

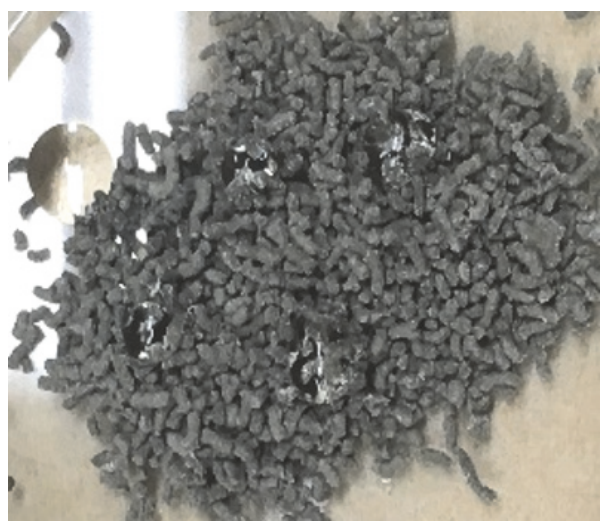

(b) After coating

FIGURE 14: Formation of wall materials according to surface tension.

for construction and added them to cementitious composites during the mixing stage. The following conclusions were derived:

(1) When cement powder was utilized as the core material, the optimal kneading agent mixing ratio for adequate consistency was obtained. However, the tests required repetition to obtain the optimal kneading agent mixing ratio, especially for core materials including substances other than cement powder or those containing at least two materials.

(2) It is considered that the test for obtaining a suitable kneading agent must be repeated because the amount of the agent used to obtain a suitable kneading consistency varies depending on the type of kneading agent. In addition, it is necessary to analyze in more detail the effects on the quality of the core material depending on the kneading agent.

(3) By applying the wall material, the core material can be protected from moisture and the strength of the surface of the capsules can be improved. However, further quantitative analysis of uniform coatings of the wall material, coating thickness, time, and effective content is necessary. 


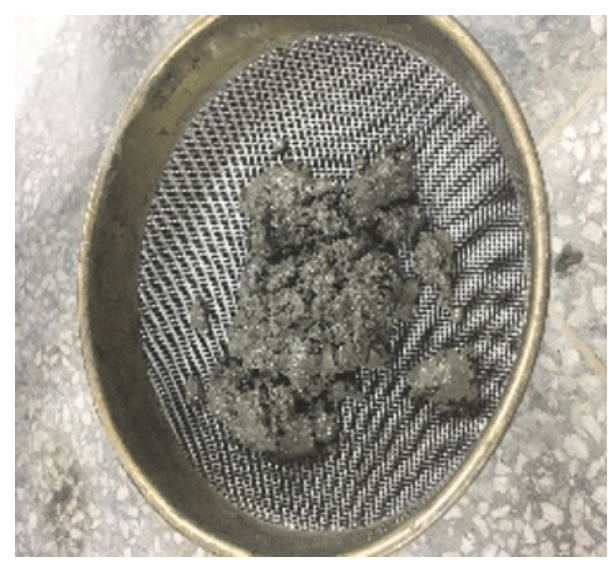

(a) Sampling

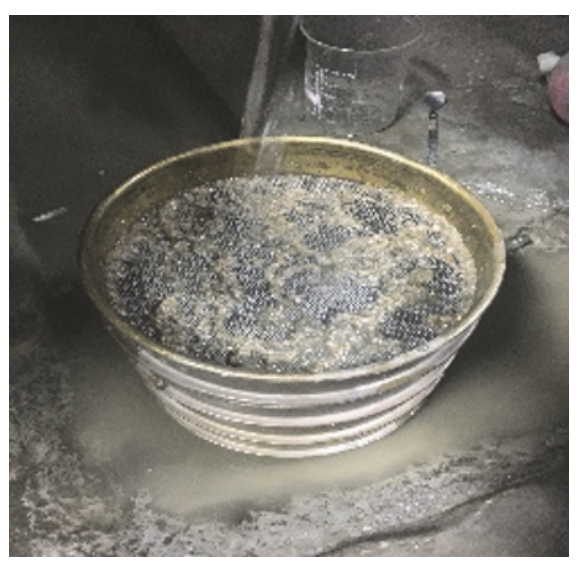

(b) Washing

Figure 15: Washing test of SHCs.

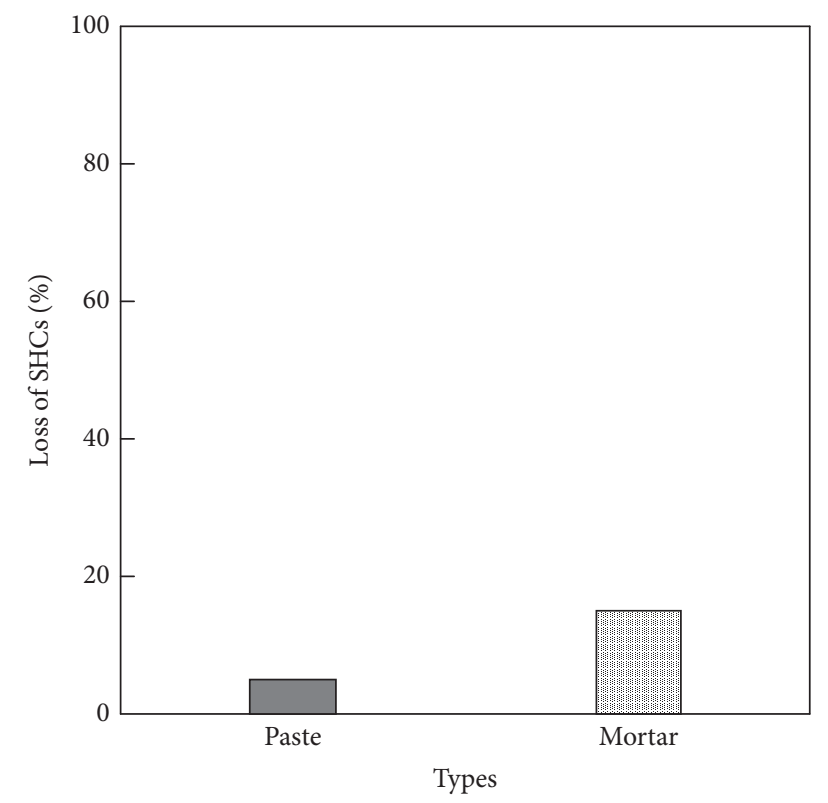

FIGURE 16: Loss of SHCs immediately after mixing with cementitious composites.

\section{Conflicts of Interest}

The authors declare that there are no conflicts of interest regarding the publication of this paper.

\section{Acknowledgments}

This research was supported by the Construction Technology Research Program funded by Ministry of Land, Infrastructure and Transport of Korean Government (Grant 17SCIPB103706-03).

\section{References}

[1] E. Schlangen and S. Sangadji, "Addressing infrastructure durability and sustainability by self healing mechanisms-recent advances in self healing concrete and asphalt," Procedia Engineering, vol. 54, pp. 39-57, 2013.

[2] E. J. An and M. S. Shin, "Healing mechanisms and assessment techniques of self-healing concrete," Proceedings of Korea Concrete Institute, vol. 26, no. 2, pp. 477-479, 2014.

[3] T. Nesterova, K. Dam-Johansen, L. T. Pedersen, and S. Kiil, "Microcapsule-based self-healing anticorrosive coatings: Capsule size, coating formulation, and exposure testing," Progress in Organic Coatings, vol. 75, no. 4, pp. 309-318, 2012.

[4] M. Hunger, A. G. Entrop, I. Mandilaras, H. J. H. Brouwers, and M. Founti, "The behavior of self-compacting concrete containing micro-encapsulated phase change materials," Cement and Concrete Composites, vol. 31, no. 10, pp. 731-743, 2009.

[5] S. A. Memon, H. Z. Cui, H. Zhang, and F. Xing, "Utilization of macro encapsulated phase change materials for the development of thermal energy storage and structural lightweight aggregate concrete," Applied Energy, vol. 139, pp. 43-55, 2015.

[6] P. S. Kim, C. K. Jo, T. H. Ju, Y. C. Choi, and J. K. Lee, "Preparation and thermal characteristics of microencapsulated PCM for none supercooling phenomenon," Proceedings of The Korean Society for Energy, vol. 2003, pp. 225-228, 2003.

[7] Y.-K. Song, Y.-H. Jo, Y.-J. Lim et al., "Sunlight-induced selfhealing of a microcapsule-type protective coating," ACS Applied Materials and Interfaces, vol. 5, no. 4, pp. 1378-1384, 2013.

[8] X. Wang, F. Xing, M. Zhang, N. Han, and Z. Qian, “Experimental study on cementitious composites embedded with organic microcapsules," Materials, vol. 6, no. 9, pp. 4064-4081, 2013.

[9] B. J. Blaiszik, N. R. Sottos, and S. R. White, "Nanocapsules for self-healing materials," Composites Science and Technology, vol. 68, no. 3-4, pp. 978-986, 2008.

[10] Korea Concrete Institute, Concrete Standard Specification, Korea Concrete Institute, Korea, 2009. 

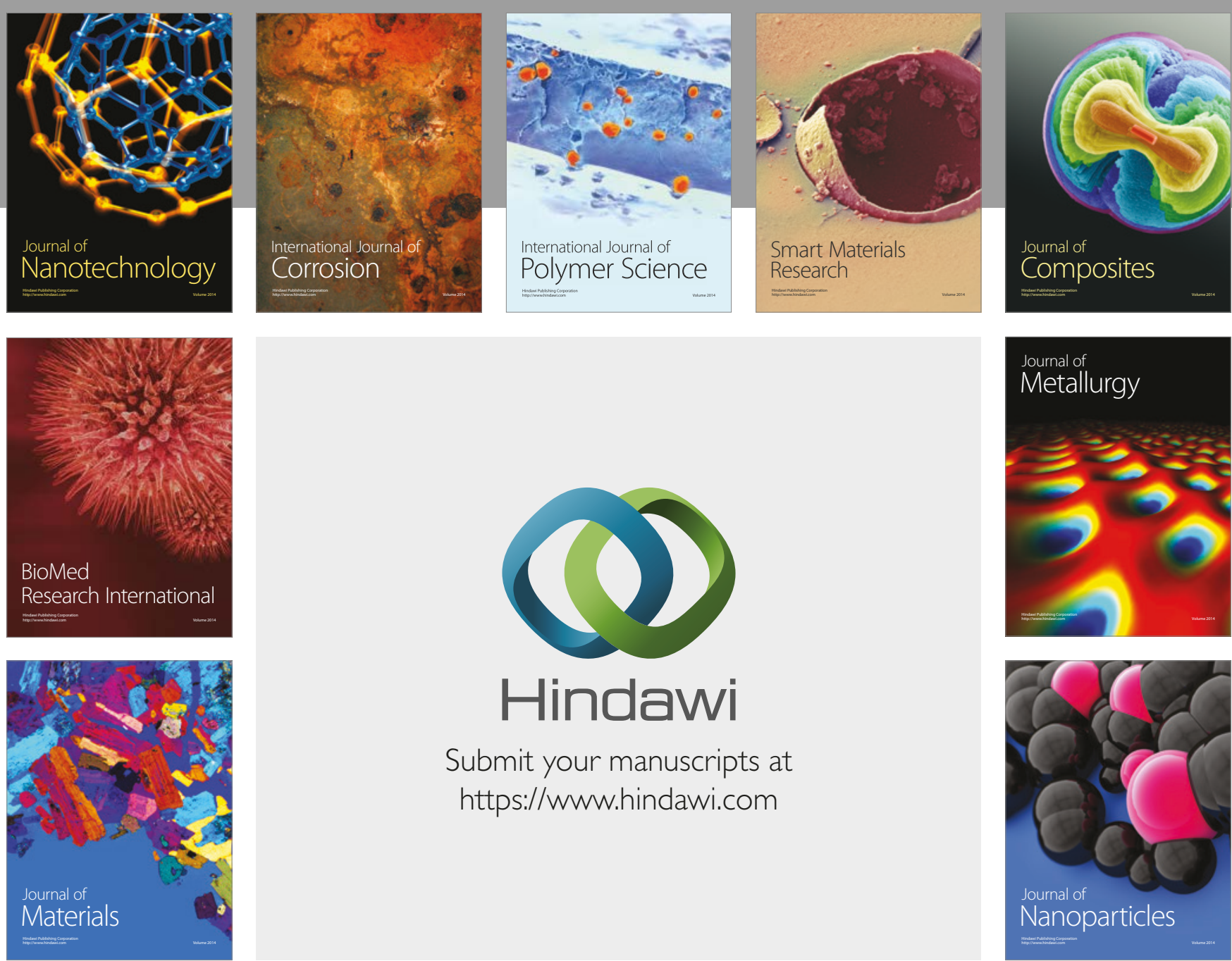

\section{Hindawi}

Submit your manuscripts at

https://www.hindawi.com
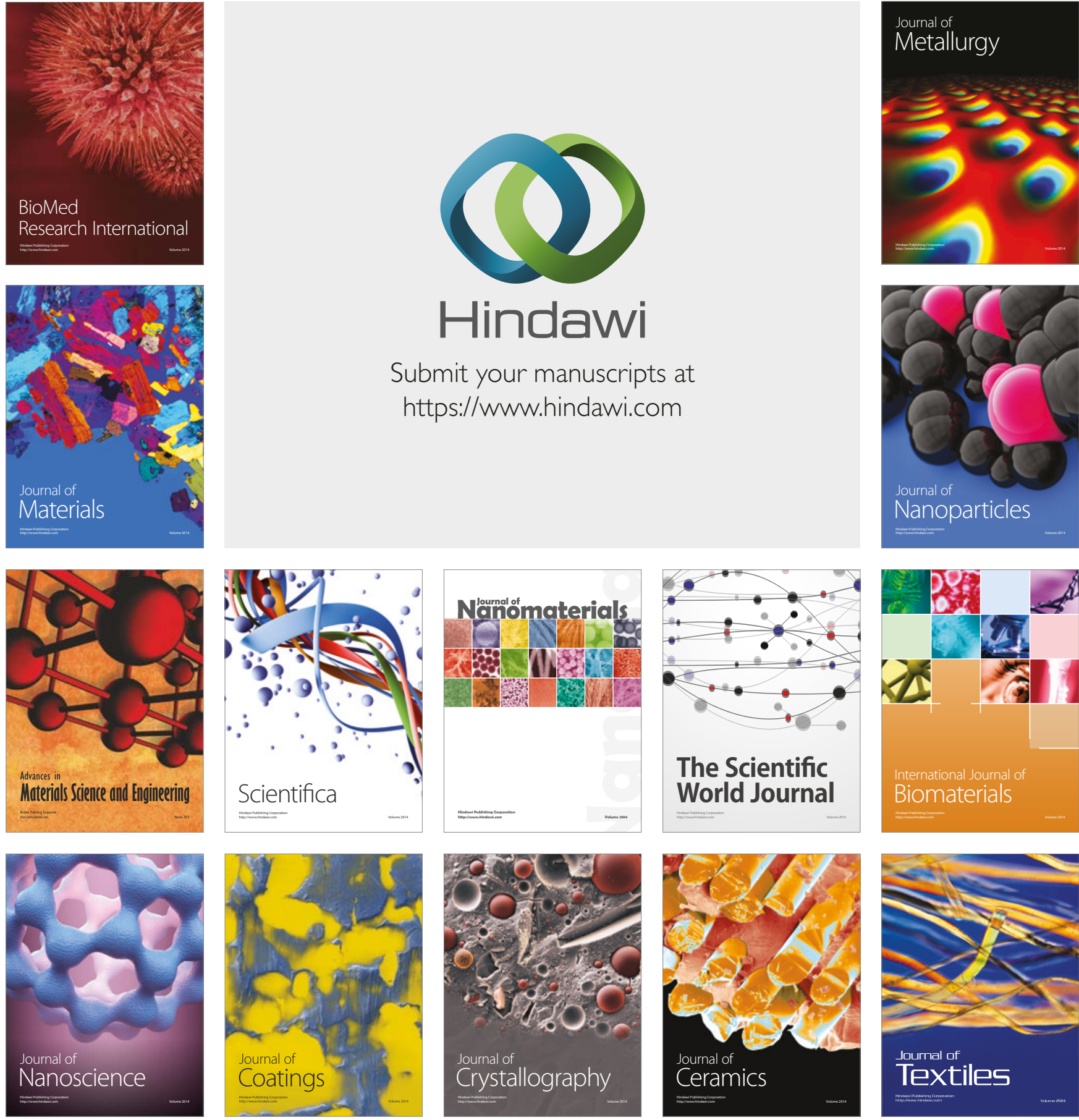

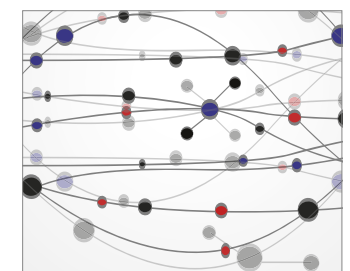

The Scientific World Journal
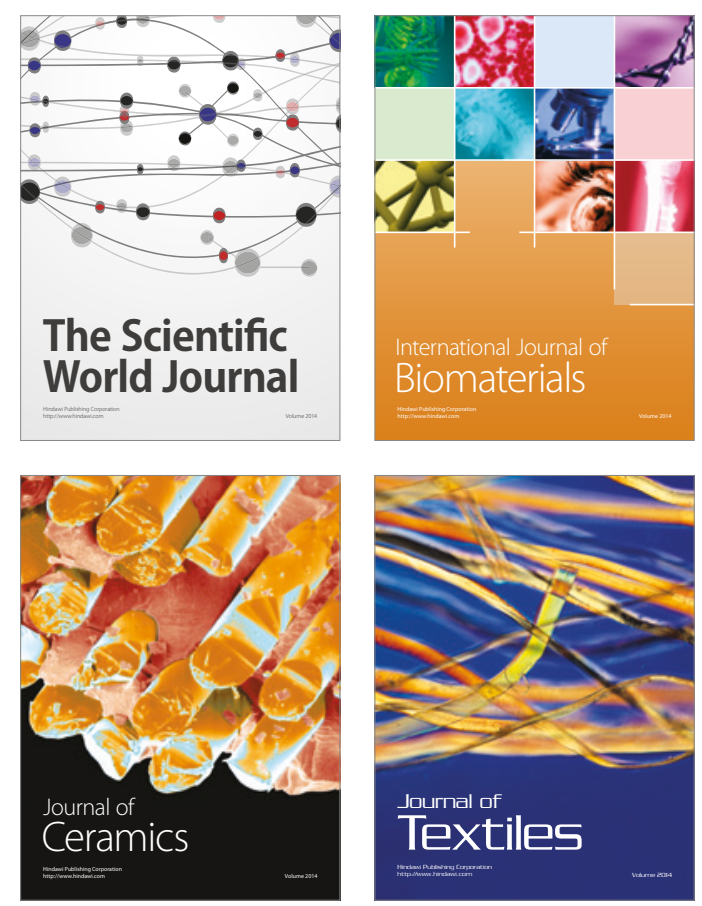